\title{
LAS CITAS DIRECTAS EN EL HABLA DE LA CIUDAD DE MÉXICO
}

\author{
QUOTATIVE MARKERS IN ORAL MEXICAN \\ SPANISH
}

\author{
Juliana de la Mora Gutiérrez \\ Universidad Autónoma de Querétaro \\ juliana.delamora@gmail.com
}

\section{Resumen}

El estudio de las citas directas en el discurso oral es uno de los terrenos más fértiles para el análisis e identificación de la variación y el cambio lingüístico (Romaine y Lange, 1991; Ferrara y Bell, 1995; Tagliamonte y Hudson, 1999). La constante incursión de estrategias innovadoras para introducir citas ha sido documentada en inglés, hebreo, alemán y japonés (Maschler, 2002; Buchstaller y D’Arcy, 2009; Buchstaller, 2013). Desafortunadamente, los estudios sobre las citas directas en el español son escasos y se limitan al español peninsular y al español de Puerto Rico. En este trabajo se presenta un análisis de los usos y distribuciones de las estrategias introductoras de citas directas, o marcos introductores de citas, en el habla de la ciudad de México. Los datos fueron extraídos del Corpus sociolingüístico de la ciudad de México (Martín y Lastra, 2011). El análisis muestra que en esta variedad dialectal predominan los verbos dicendi y los marcadores nulos como estrategias para introducir el discurso directo. Asimismo, revela la incursión de nuevas formas que no habían sido documentadas en este dialecto. Finalmente, el análisis de la distribución social de estas formas muestra que es un sistema socialmente estratificado. Si bien no se cuenta con ningún estudio comparativo que permita establecer parámetros en tiempo real, los datos en tiempo aparente aquí presentados sugieren que el sistema de citas directas en el español de México está en proceso de cambio lingüístico.

Palabras Clave: citas, discurso directo, verbos dicendi, español de México, sociolingüística hispánica

\section{Abstract}

The study of quotative markers is one of the most recurrent places for the analysis of language variation and change (Romaine and Lange, 1991; Ferrara and Bell, 1995; Tagliamonte and Hudson, 1999). The constant incursion of innovative forms has been documented in English, Hebrew, German and Japanese (Maschler, 2002; Buchstaller and D’Arcy, 2009; Buchstaller, 2013). Unfortunately, the studies of quotative markers are still scarce in Spanish and are limited to Peninsular and Puerto Rican dialects. In this paper, I present an analysis of the uses and distribution of quotative markers in oral Mexican Spanish. Data

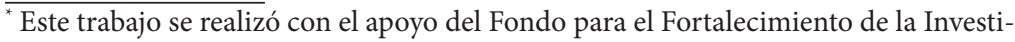
gación (FOFI-UAQ), de la Universidad Autónoma de Querétaro.
} 
was extracted from the Corpus Sociolingüístico de la ciudad de México (Martín and Lastra, 2011). The analysis shows that the most frequent markers are verbs of communication and null forms. It also shows the incursion of new markers, not previously documented in this dialect. Finally, the analysis of the social distribution of the incoming forms suggest that the quotative markers' system is socially stratified. It is still necessary to establish parameters in real time, however the data from apparent time presented in this paper suggest a change in progress.

KEYWORDS: quotatives, direct discourse, Mexican Spanish, Spanish sociolinguistics

FECHA DE RECEPCIÓN: 29/03/2018

FECHA DE ACEPTACIÓN: 12/06/2018

\section{Introducción}

El análisis de las citas directas en el discurso oral ha sido uno de los terrenos más fértiles para el estudio de la variación y el cambio lingüístico (Romaine y Lange, 1991; Ferrara y Bell, 1995; Tagliamonte y Hudson, 1999). Una gran parte de los estudios ha documentado que los sistemas de citas directas (quotative markers) están socialmente estratificados siendo el locus ideal para el estudio de la variación sociolingüística (Buchstaller y D’Arcy, 2009; Buchstaller et al., 2010; Tagliamonte y D'Arcy, 2004; Tagliamonte y Hudson, 1999). Además, diversos estudios en lenguas tipológicamente diferentes como el inglés, hebreo, alemán y japonés (Buchstaller, 2013; Maschler, 2002) han mostrado una constante incursión de formas innovadoras, algunas de las cuales se han mantenido en variación estable y otras muestran patrones de cambio lingüístico en progreso. Adicionalmente, se ha documentado que los patrones de incorporación de nuevas formas siguen procesos muy similares, lo cual sugiere que el sistema de citas directas es un sistema regido por factores lingüísticos específicos.

A pesar de la gran cantidad de datos que puede aportar el estudio de los introductores de citas directas en el campo de la variación y el cambio lingüístico, y de la gran cantidad de tra- 
bajos que se han hecho en otras lenguas, particularmente en el inglés, estos trabajos aún son escasos en el español y en el caso de la variante dialectal mexicana, el único estudio encontrado en la bibliografía analiza datos de los años sesenta. Así, el objetivo fundamental de este trabajo es documentar los usos y la distribución de las estrategias introductoras de citas directas en el discurso oral a partir de datos del español de la ciudad de México. Se pretende también identificar los patrones de uso en función de factores externos, identificar si hay formas innovadoras y determinar si hay patrones de estratificación social que sean indicadores de cambio lingüístico en progreso o variación estable (Labov, 1963, 1966). Igualmente, a partir del uso y la distribución general de los marcadores de citas del español de México se busca determinar las diferencias y similitudes que existen con los sistemas de marcadores de citas de otros dialectos del español que han sido documentados.

\section{Las citas directas en el español. Del discurso a la variación}

La capacidad de reproducir un discurso es un universal del lenguaje que ha sido ampliamente estudiado y descrito desde diversas disciplinas. Los estudios lingüísticos se han centrado en dos mecanismos fundamentales: el discurso directo y el discurso indirecto. A pesar de la gran tradición en el estudio de estos mecanismos no existe consenso en las definiciones de cada uno de ellos. Una de las diferencias fundamentales entre las citas directas e indirectas se da "en el modo en el que se presentan las palabras atribuidas a otra fuente: en la CD el hablante actual presenta las ideas como palabras de hablantes distantes de su marco espacio-temporal; en la CI, por el contrario, las ideas se ajustan para encajarlas en el marco espacio-temporal del hablante actual" (Camargo, 2012: 3).

En este trabajo se considera que las citas directas son un tipo de demostración que permite ilustrar algo mediante la ejemplificación (Clark y Gerrig, 1990: 765). De esta manera se asume que al introducir una cita directa los referentes no se describen, sino que se representan. En cuanto a su estructura, Mal- 
donado sugiere que el discurso directo "está constituido por una 'expresión introductora' (EI) que contiene un verbo de 'decir' flexionado y una 'cita directa' (CD) marcada tipográficamente por guiones o comillas y que es siempre la reproducción literal de un enunciado" (1999: 3554). Cabe señalar que en este trabajo se utilizan de manera intercambiable los términos 'expresión introductora', 'estrategia introductora', 'marco introductorio' y 'marcador de citas' para referirse a las formas lingüísticas usadas por los hablantes para introducir y enmarcar una cita directa.

Con respecto a la literalidad de las citas, Maldonado (1999) reconoce que el discurso citado puede o no ser literal y puede ser real o imaginario, ya que es parte de un diálogo reconstruido como lo plantea Tannen (1986). En cuanto a las características discursivas de las citas en los diálogos reconstruidos, se ha sugerido que deben formar una unidad dialogal en la que las intervenciones se corresponden con voces y en orden diferentes tales como secuencias en pares de pregunta/respuesta o de aserción/ intervenciones reactivas (Briz, 2006: 46). Así, en este trabajo se asume que las citas directas representan la reconstrucción, de forma aproximada y no literal, de diálogos pasados, posibles e imaginarios que se atribuyen a una fuente (Camargo, 20072008: 49).

Una de las estrategias introductoras del discurso directo que frecuentemente se observa en las lenguas es el uso de los verbos dicendi, o verbos de comunicación, como se observa en (1) y (2):

(1) Jim said 'I love you'

(2) entonces ella dijo/ "no/ pues < pus $>$ nos vamos al departamento nuevo...” (CSCM, 13)

Además de estas formas, comúnmente descritas y reconocidas por las gramáticas, se ha documentado en el inglés la rápida incursión de nuevas estrategias para introducir citas directas (Romaine y Lange, 1991; Ferrara y Bell, 1995; Tagliamonte y Hudson, 1999; Tagliamonte y D’Arcy, 2004; Buchstaller y D’Arcy, 2009; Buchstaller et al., 2010). Dentro de estas estra- 
tegias se encuentra el uso de formas como be all, go y be like, ilustrada en (3)

(3) My daughter's like 'Mommy can I help you with the laundry?' $\varnothing$ 'Of course you can' (Buchstaller, 2013: 7)

El creciente interés en el discurso reportado ha permitido constatar que la variación observada en estos sistemas está social y lingüísticamente determinada: hay estratificación social, rápida y constante incursión de nuevas formas y evidencias sobre procesos de gramaticalización. En el caso del español, también ha sido documentada la incursión de nuevos introductores de citas directas, tal es el caso de los marcadores nulos o freestanding forms y los marcadores denominados $\mathrm{Y}(\mathrm{FN})$ conformados por una conjunción y una frase nominal (Cameron, 1998), ilustradas en (4) y (5) o las citas introducidas por "así como que" como en (6):

(4) y éramos como cinco/ habíamos llegado/Ø "oiga maestro" (CSCM 13)

(5) Y ella, "Ah no, mi'jo" (Cameron, 1998: 49)

(6) Jenny: Hablan como, así como que “iyeehah!” (Holguín, 2015: 59)

A pesar de que las formas innovadoras han sido documentadas ya en español, aún son pocos los trabajos que han investigado los usos y posible estratificación social de las citas directas $\mathrm{y}$ aún menos los que han investigado los patrones de variación y cambio lingüístico en dichos sistemas. Además, las variedades dialectales que han sido estudiadas no han contemplado el estudio del español de México.

Los estudios sobre las citas directas en el español se enmarcan en tres líneas de investigación: por un lado, encontramos los análisis de corte discursivo, cuyo interés fundamental es contrastar los usos y las frecuencias del estilo directo e indirecto. En este tipo de trabajos también se presentan descripciones detalladas de los usos de ambas variables en función de factores sociales como la edad, el género y el nivel educativo de los hablantes (Ma- 
teus, 2005; Camargo, 2007-2008; García, 2009; Gallucci, 2009, 20012a, 2012b, 2013, 2014; Fernández, 2012; Gallucci y Vargas, 2015). Por otro lado, encontramos trabajos en los que se estudian de forma secundaria los marcadores de citas, como en el caso de Estellés-Arguedas (2015) en el que presenta un análisis de los patrones entonativos del discurso directo $y$, finalmente, los estudios variacionistas que además de describir las frecuencias y distribuciones de los marcadores de citas directas en función de factores internos y externos, buscan explicar los efectos de estos últimos en la variación de los usos de los marcadores (Cameron, 1998).

Dentro de los análisis discursivos del discurso reportado encontramos una gran cantidad de trabajos sobre el español de Venezuela. Muchos de estos trabajos se han centrado en el contraste entre el estilo directo e indirecto, aunque también presentan descripciones detalladas de la distribución de las formas que introducen las citas directas considerando factores sociales como la edad y el género de los hablantes. En el caso de la frecuencia de las citas directas e indirectas todos los trabajos coinciden en la prevalencia de las primeras sobre las segundas (Mateus, 2005; Gallucci, 2009; García, 2009; Fernández, 2012; Gallucci y Vargas, 2015). La forma más frecuente para introducir citas directas es mediante verbos dicendi (Gallucci, 2009, 2013, 2014; Mateus, 2005; García, 2009; Fernández, 2012; Gallucci y Vargas, 2015). Con respecto a la persona gramatical se ha documentado que las citas directas son más frecuentes en primera persona (Gallucci, 2009; Gallucci y Vargas, 2015) y los usos de las citas directas son más frecuentes en mujeres que en hombres (Gallucci, 2013). Las tres funciones más comunes de las citas directas son relatar, ejemplificar y argumentar (Gallucci, 2013; Fernández, 2012; Galluci y Vargas, 2015). Además de los verbos dicendi, Gallucci y Vargas documentaron otras 3 estrategias para introducir citas directas: marcos nulos, (Y) $\mathrm{SN}$, marcador discursivo. Las formas dicendi fueron las más frecuentes, seguidas de los marcadores nulos, $\mathrm{Y}(\mathrm{SN}) \mathrm{y}$, finalmente, las citas introducidas por marcadores discursivos.

Además de los estudios de corte discursivo, encontramos trabajos que indagan en otros aspectos, como en el caso de Es- 
tellés-Arguedas (2015). La autora analiza la relación entre los cambios en la entonación y la marcación de la evidencialidad en el discurso directo en español. Sus resultados revelan, por un lado, que al igual que en otros dialectos, la forma que predomina para introducir el discurso directo son los verbos dicendi (69\%) seguidos por las formas nulas (16\%), además muestra que ambas estrategias tienen mecanismos entonativos similares, aunque en el caso de las formas nulas, los cambios en la entonación son obligatorios.

Encontramos también trabajos que analizan detalladamente los usos y frecuencias de los introductores de citas directas en jóvenes hablantes de dos lenguas distintas (Palacios, 2013) considerando factores internos y externos. Palacios presenta un análisis comparativo en dos grupos de adolescentes, uno compuesto por hablantes de inglés y otro por hablantes de español peninsular. En el caso del inglés, los resultados muestran que las formas dicendi corresponden únicamente al 29\% de los datos, mientras que el $71 \%$ restante está conformado por formas innovadoras (go, be like, say like, go like). En el caso de los adolescentes españoles, el patrón es completamente inverso; el 69\% de los introductores de citas directas son verbos dicendi, el $8 \%$ 'marcador nulo', 11\% las formas ' $\mathrm{Y}(\mathrm{FN})$ ' y el 12\% restante corresponde a otras formas. En el caso del español de México, van der Houwen (1998), muestra que las formas de cita directa en esta variedad dialectal son más frecuentes que la cita indirecta. Las formas más frecuentes para introducir citas directas son los verbos dicendi y el tiempo predominante fue el presente. Además, fue más común encontrar que los verbos dicendi no estuvieran acompañados de un sujeto explícito.

La tercera línea de investigación de los marcadores de citas está compuesta por los trabajos de corte variacionista que buscan determinar cuál es el rol de los factores sociales y lingüísticos en los usos variables de estas variantes. El único trabajo encontrado que se enmarca en esta línea es el de Cameron (1998) sobre el español de Puerto Rico. Los porcentajes de distribución de los introductores de citas directas muestran que la forma más común es el uso de verbos dicendi (62\%), seguido por los nu- 
los $(25 \%)$ y, finalmente, las formas Y(FN) (13\%). Además del predominio de los verbos dicendi sobre otras formas, Cameron encontró que la edad fue uno de los factores sociales que determinaba el uso variable de los marcadores de citas directas. En el caso de los verbos dicendi y las formas (Y)FN, Cameron mostró que los hablantes mayores favorecen el uso de los verbos dicendi y desfavorecen las formas (Y)FN. Al igual que Gallucci (2009), Cameron muestra que las formas de primera persona son las más recurrentes. Para los factores internos, los resultados mostraron que el tipo de cláusula, la persona, el número gramatical y la animacidad fueron estadísticamente significativos en la variación.

En el cuadro 1, se presenta un resumen de los usos y distribuciones de los marcos introductores de citas directas en tres dialectos del español. Como se puede observar, predominan los verbos dicendi seguidos por los 'marcadores nulos'. Cabe resaltar que las formas dicendi y los 'marcadores nulos' suman entre el 75\% y el 93\% de las estrategias utilizadas para introducir citas directas en los cuatro dialectos. La distribución de las otras formas es difícilmente comparable ya que en algunos casos los autores únicamente hacen la distinción entre verbos de comunicación y otros verbos, en otros casos las categorías establecidas entre autores no coinciden, los datos no son lo suficientemente explicativos o simplemente no se incluyen en el análisis las citas introducidas por otros marcadores.

\begin{tabular}{|l|c|c|c|c|c|}
\hline & $\begin{array}{c}\text { Puerto Rico } \\
\text { (San Juan) } \\
\text { (Cameron, } \\
1998)\end{array}$ & $\begin{array}{c}\text { Venezuela } \\
\text { (Mérida) } \\
\text { (Fernández, } \\
2012)\end{array}$ & $\begin{array}{c}\text { Venezuela } \\
\text { (Caracas) } \\
\text { (Gallucci } \\
\text { y Vargas, } \\
2015)\end{array}$ & $\begin{array}{c}\text { España } \\
\text { (Valencia) } \\
\text { (Estellés- } \\
\text { Arguedas, } \\
2015)\end{array}$ & $\begin{array}{c}\text { México } \\
\text { (Cd. México) } \\
\text { (van der } \\
\text { Houwen, } \\
1998)\end{array}$ \\
\hline Dicendi & $62 \%$ & $68 \%$ & $53 \%$ & $71 \%$ & $75 \%$ \\
\hline Nulo & $25 \%$ & $32 \%$ & $40 \%$ & $16 \%$ & -- \\
\hline Y(FN) & $13 \%$ & -- & $4 \%$ & -- & -- \\
\hline Otro & -- & -- & $3 \%$ & $13 \%$ & $25 \%$ \\
\hline
\end{tabular}

Cuadro 1. Uso y distribución de las citas directas en algunos dialectos del español 
A pesar de que los estudios sobre el discurso directo en el español oral aún son escasos, se observa en todos los trabajos una alta frecuencia de verbos dicendi e introductores 'nulos' los cuales corresponden en la mayoría de los casos a más del $80 \%$ de los datos, en oposición a lenguas como el inglés en las que se observa una notable disminución en el uso de estas formas. Además, los trabajos previos también han mostrado que el uso de las citas directas está estratificado socialmente, con efectos considerables de la edad (Cameron, 1998; Palacios, 2013; Estellés-Arguedas, 2015). Finalmente, muestran que en el español hay mecanismos alternativos para introducir citas (Cameron, 1998; Palacios, 2013; Estellés-Arguedas, 2015; Gallucci y Vargas, 2015; Holguín, 2015) y formas innovadoras y dialectos poco estudiados como es el caso del español de México.

\section{Metodología}

\subsection{Corpus y entrevistas}

Los datos analizados en este estudio provienen del Corpus sociolingüístico de la ciudad de México (CSCM) (Martín y Lastra, 2011). ${ }^{1}$ Dicho corpus está conformado por 108 entrevistas. Para el análisis aquí presentado se analizaron ejemplos de 54 entrevistas, seleccionadas por muestreo de cuotas, considerando 2 géneros $\mathrm{x} 3$ grupos de edad $\mathrm{x} 3$ niveles de instrucción $\mathrm{x} 3$ informantes por casilla. El cuadro 2 ilustra el número de entrevistas seleccionadas por casilla.

\begin{tabular}{|l|c|c|c|c|c|c|}
\hline & \multicolumn{2}{|c|}{ Escolaridad baja } & \multicolumn{2}{c|}{ Escolaridad media } & \multicolumn{2}{c|}{ Escolaridad alta } \\
\hline & Hombre & Mujer & Hombre & Mujer & Hombre & Mujer \\
\hline Jóvenes & 3 & 3 & 3 & 3 & 3 & 3 \\
\hline Adultos & 3 & 3 & 3 & 3 & 3 & 3 \\
\hline Mayores & 3 & 3 & 3 & 3 & 3 & 3 \\
\hline
\end{tabular}

Cuadro 2. Entrevistas seleccionadas en la muestra analizada

\footnotetext{
${ }^{1}$ El corpus utilizado forma parte del Proyecto para el Estudio Sociolingüístico de España y América (PRESEEA) cuya metodología se describe en Moreno (1997 y 2003). Para la metodología de clasificación y análisis del discurso reportado ver Camargo (2012).
} 


\subsection{Extracción, codificación y análisis de datos}

A partir de las 54 entrevistas seleccionadas, se realizó la extracción, codificación y análisis de las estrategias introductoras de citas directas utilizadas por los informantes. Las producidas por los entrevistadores no fueron consideradas en este análisis debido a que no se cuenta con sus datos sociolingüísticos. Otro de los criterios de inclusión fue que la cita directa tuviera el contexto discursivo necesario para poder codificarla. A partir de estos criterios, se trabajó con un total de 4,632 ejemplos. Todos los ejemplos extraídos de las entrevistas se codificaron a partir de variables sociales y variables lingüísticas.

\subsubsection{Variables sociales}

Las variables sociales consideradas fueron la edad, el nivel educativo y el sexo de los hablantes. Para establecer las categorías de cada una de estas variables, se siguió el criterio utilizado por Martín y Lastra $(2011,2012,2015)$ en el Corpus sociolingüístico de la ciudad de México:

a) Edad. Para el grupo de edad, se consideran tres generaciones: los jóvenes, formada por personas que tienen entre 20 y 34 años; la generación de adultos conformada por hablantes de entre 34 y 54 años; la generación de mayores, cuyos hablantes tienen 55 años o más.

b) Nivel educativo. El nivel educativo de los hablantes se divide en bajo, medio y alto. En el nivel bajo se clasifican los hablantes que han recibido como máximo educación primaria, el nivel medio se conforma por hablantes que han recibido enseñanza intermedia, hasta secundaria o bachillerato. Finalmente, el nivel educativo alto corresponde a informantes que recibieron enseñanza superior, universitaria o técnica.

c) Género

\subsubsection{Variables lingüísticas}

Dentro de las variables lingüísticas se codificó para cada ejemplo la estrategia utilizada por el hablante para introducir las citas 
directas. Los tipos de introductores encontrados se clasificaron en 'dicendi', 'marcador nulo, '(Y)FN', 'así', 'o sea', 'SER de que' y 'otros' atendiendo a los siguientes criterios: ${ }^{2}$

1. Dicendi. Todas las citas directas introducidas por un verbo dicendi:

(7) entonces/ yo le decía a mi mamá/ "es que necesito para un libro" (CSCM, 8)

(8) le hablo a su mamá/ "entiéndete con tu madre/ y mejor ahí muere" (CSCM, 16)

(9) entonces ya// agarra y me dice/"ahorita te paso la paciente"/ dice/“es más/ yo te voy a ayudar" (CSCM, 10)

2. Marcador nulo. ${ }^{3}$ No hay marca lingüística que introduzca la cita, sin embargo hay un cambio de entonación y una breve pausa que permiten identificar el discurso reportado:

(10) ya cuando se nos estaba acabando [el este] el dinero/ Ø "oye pues $<\sim$ pus $>$ tenemos que hacer algo porque aquí nos estamos muriendo de hambre" (CSCM, 16)

(11) y éramos como cinco/ habíamos llegado/ "oiga maestro/ es que la ciudad está derrumbada"/ Ø "no me interesa/ vamos a hacer el examen" (CSCM, 13)

3. (Y)FN. En esta categoría se incluyeron todas las citas directas introducidas por una frase nominal, léxica o pronominal, opcionalmente acompañada por la conjunción (Y) (ver Cameron, 1998):

(12) y yo/ “'historia?/ ¿y existe esa carrera?” (CSCM, 18)

(13) comimos crepas/ unas crepas riquísimas pero ¡enormes < enor::mes>!/nosotros/ "queremos una chica"/ no hombre/una

\footnotetext{
${ }^{2}$ La posición del verbo dicendi ha sido estudiada en algunas variedades del español (Gallucci, 2013), a pesar de que se reconoce la importancia de la posición del verbo dicendi como un factor notable para la variación, para los fines de este trabajo únicamente se incluyen en esta categoría los casos en los que el verbo dicendi aparece antes de la cita.

${ }^{3}$ Estudios como los de Kvavik (1986), Cabedo (2007) y Estellés-Arguedas (2015) han mostrado que el discurso directo puede introducirse en la conversación mediante un cambio en el tono y un patrón de entonación particular que es obligatorio en las citas que no tienen una marca que las introduce, como en este caso el 'marcador nulo'.
} 
grandísima/ y nosotros/ “¡no/ ya no nos traiga las otras dos! nada más una" (CSCM, 10)

4. Marcador 'así (así de (que), así como)'

(14) las ves y llegan así de "doctora/ sí se pudo/ ya estoy súper bien/ este ya me pinto/ ya como/ ya esto/ ya lo otro" (CSCM, 12)

(15) entonces lo grabé en un casete/ tres veces/ para que/los niños trabajaran// y ya/ ese día sí fue así como que/ "bueno/ pues < pus> ahora sí/ pon el disco porque/ se oye más bonito" (CSCM, 44)

5. Marcador 'o sea'

(16) yo/trataba de hacer amistades y ganaba muchas amistades/o sea/ "sí/ hola/ ¿cómo estas?"/ siempre charlaba y sacaba una/ tontería y sacaba plática/ (CSCM, 3)

(17) o sea/ "esta persona hizo esto/ y a mí me hizo sentir/ o me dio la impresión de esto" (CSCM, 24)

6. 'SER de que' (CSCM, 24)

(18) entonces/ al momento de llegar aquí a la Enah/ era de que// "ay mamá/ tengo que salir de práctica" (CSCM, 11)

(19) no es de que/ ahí "voy" y me tardo diez minutos en bajar (CSCM, 11)

7. Otros marcadores

(20) y éramos como cinco/ habíamos llegado/ "oiga maestro/ es que la ciudad está derrumbada"/ "no me interesa/ vamos a hacer el examen"

(21) ves a alguien te vas te arrimas $\mathrm{y}<\sim \mathrm{y}:>/$ “iquihúbole $<\sim$ quehóbole>!!" (risa) (CSCM, 37)

(22) me ha tocado gente que se sube y este/ "voy a tal parte"/ sí/ “¿cuánto me cobra?” (CSCM, 80)

\section{Resultados}

\subsection{Distribución general}

La distribución general de los marcadores de citas identificados en el español de la ciudad de México se presenta en el cuadro 3. Estos datos muestran que, en el español de la ciudad de México, al igual que en otras variedades dialectales, predomina el uso de verbos 'dicendi' como mecanismo para introducir la cita (63\%) seguido del 'marcador nulo' (28\%), ambos marcadores confor- 
man el 91\% del total de los datos. El 9\% restante corresponde a otros marcadores, algunos de ellos ya documentados en otras variedades del español. Debido a que tanto las formas introducidas por verbos dicendi, como las formas nulas, son las más frecuentes y las que se encuentran en todas las variedades del español, nos referiremos a ellas de aquí en adelante como 'formas canónicas'. Por el contrario, los marcadores que conforman el 9\% restante de los datos serán denominados 'formas no canónicas'. Además, esta distinción permitirá distinguir las formas previamente documentadas de las posibles formas innovadoras. Dentro de las formas no canónicas predominaron los marcadores clasificados como 'otros' (4\%), seguidos por las formas '(Y) FN' (2\%), en seguida las citas introducidas por 'asî' (2\%) y, finalmente, los marcadores 'SER de que' y 'o sea' con porcentajes de $.8 \%$ y $.2 \%$ respectivamente.

\begin{tabular}{|l|c|c|}
\hline \multicolumn{1}{|c|}{ Marcador } & Porcentaje & $\mathrm{n}$ \\
\hline Dicendi & $63 \%$ & 2926 \\
\hline Nulo & $28 \%$ & 1293 \\
\hline Otro & $4 \%$ & 195 \\
\hline (Y)FN & $2 \%$ & 97 \\
\hline Así & $2 \%$ & 70 \\
\hline SER de que & $0.8 \%$ & 38 \\
\hline O sea & $0.2 \%$ & 13 \\
\hline TOTAL & $100 \%$ & $\mathrm{~N}=4632$ \\
\hline
\end{tabular}

Cuadro 3. Distribución de los marcadores de citas directas en el español de México

La distribución de los marcadores canónicos (dicendi y 'marcador nulo') y de los marcadores no canónicos ('otro', 'Y(FN), 'así', 'SER de que,' 'o sea') se presenta en el cuadro 4:

\begin{tabular}{|c|c|c|}
\hline Marcador & Porcentaje & $\mathrm{n}$ \\
\hline Canónicos & $91 \%$ & 4219 \\
\hline No canónicos & $9 \%$ & 413 \\
\hline Total & $100 \%$ & $\mathrm{~N}=4632$ \\
\hline
\end{tabular}

Cuadro 4. Distribución de los marcadores canónicos y no canónicos en el español de México 
Si bien el porcentaje de las formas no canónicas en el español de México no alcanza ni siquiera el 10\% del total de los datos, se puede constatar que hay una gran variedad en estas formas e incluso algunas de ellas solo aparecen en determinados grupos etarios como veremos en secciones posteriores. Además, estos marcadores no canónicos no habían sido documentados anteriormente en el español de México (ver van der Houwen, 1998), y presentan, en su mayoría, patrones de estratificación.

\subsection{Marcadores canónicos}

En esta sección se presentan los resultados del análisis de los marcadores dicendi y marcadores nulos por grupos de edad, nivel educativo y género.

\subsubsection{Marcadores canónicos por grupo de edad}

La distribución de las citas introducidas por verbos 'dicendi' y 'nulo', o marcadores canónicos, por grupo de edad se presenta en el cuadro 5. Los resultados muestran nuevamente una predilección por los verbos 'dicendi' en los tres grupos, utilizados por los jóvenes en el 76\% de los ejemplos, por los adultos el $71 \%$ y por los mayores el $61 \%$. En el caso de la forma nula, son los hablantes mayores quienes presentan una frecuencia más alta (39\%) seguida por los adultos (29\%) y, finalmente, los jóvenes (24\%).

\begin{tabular}{|l|c|c|c|}
\hline & Joven & Adulto & Mayor \\
\hline Dicendi & $76 \%(953)$ & $71 \%(1120)$ & $61 \%(853)$ \\
\hline Nulo & $24 \%(309)$ & $29 \%(450)$ & $39 \%(534)$ \\
\hline Total & $100 \%(1262)$ & $100 \%(1570)$ & $100 \%(1387)$ \\
\hline
\end{tabular}

Cuadro 5. Distribución de marcadores canónicos por grupo de edad

\subsubsection{Marcadores canónicos por nivel educativo}

Los resultados por nivel educativo muestran una distribución bastante similar entre los tres grupos (cuadro 6). En los hablantes de los tres niveles, el introductor de citas directas preferido es el verbo dicendi, con una distribución del $67 \%$ en hablantes de 
nivel alto, $68 \%$ para los hablantes de nivel medio y $72 \%$ para los de nivel bajo. En el caso del 'marcador nulo' es utilizado por los hablantes de nivel alto en el 33\% de los ejemplos, en 32\% por los hablantes de nivel medio y $28 \%$ por los hablantes de nivel bajo.

\begin{tabular}{|l|c|c|c|}
\hline & Alto & Medio & Bajo \\
\hline Dicendi & $67 \%(730)$ & $68 \%(942)$ & $72 \%(1254)$ \\
\hline Nulo & $33 \%(354)$ & $32 \%(452)$ & $28 \%(487)$ \\
\hline Total & $100 \%(1084)$ & $100 \%(1394)$ & $100 \%(1741)$ \\
\hline
\end{tabular}

Cuadro 6. Distribución de marcadores canónicos por nivel educativo

Si bien la diferencia entre los tres niveles no es muy grande, se observa que los grupos medio y alto comparten una distribución similar para ambas formas. Asimismo, se observa que en el caso de los hablantes de nivel bajo hay un uso menor del 'marcador nulo' y un uso mayor, en comparación con los otros grupos, del marcador dicendi.

La distribución de usos de los marcadores no canónicos por nivel de escolaridad muestra una ligera preferencia por la forma dicendi en los hablantes de nivel bajo y en el caso de los hablantes de nivel alto y medio por el uso del 'marcador nulo'.

\subsubsection{Marcadores canónicos por sexo}

En el caso de los marcadores canónicos por sexo (cuadro 7) se observan datos sumamente interesantes. Mientras que el uso del verbo dicendi en las mujeres corresponde al $75 \%$ de los casos, en los hombres esta misma forma tiene un porcentaje menor correspondiente al $61 \%$.

\begin{tabular}{|l|c|c|}
\hline & Mujeres & Hombres \\
\hline Dicendi & $75 \%(1867)$ & $61 \%(1059)$ \\
\hline Nulo & $25 \%(611)$ & $39 \%(682)$ \\
\hline \multicolumn{1}{|c|}{ Total } & $100 \%(2478)$ & $100 \%(1741)$ \\
\hline
\end{tabular}

Cuadro 7. Distribución marcadores canónicos por sexo

La disminución en el uso de los verbos dicendi en los hablantes hombres parece estar compensada por un mayor uso de 
las formas nulas. La frecuencia del marcador nulo es mayor en el grupo de los hombres con 39\% de los ejemplos, comparado con el uso de las mujeres en el que la misma forma aparece solamente en el $25 \%$ de los ejemplos. En el caso del sexo, los datos muestran una mayor frecuencia de uso del marcador dicendi en las mujeres que en los hombres.

\subsection{Marcadores no canónicos}

En esta sección se presenta el análisis de los marcadores 'así, 'SER de que', '(Y)FN', 'o sea' y 'otros', cruzados con las variables sociales grupo de edad, nivel educativo y sexo.

\subsubsection{Marcadores no canónicos por grupo de edad}

Con respecto al análisis de la distribución y las frecuencias de las formas no canónicas por grupo de edad se encontró que salvo en el caso de la forma '(Y)FN', en todos los demás marcadores la distribución de las formas no canónicas está estratificada según el grupo etario (cuadro 8).

Llama la atención también que es en el grupo de los jóvenes en donde aparece la mayor variedad de formas no canónicas y con una distribución muy pareja, con excepción del marcador 'o sea', cuyos porcentajes corresponden al 7\% de los usos de los marcadores no canónicos. A pesar de ello, es en el grupo de hablantes jóvenes en el que aparece con mayor frecuencia la forma 'o sea'.

El marcador 'así' muestra frecuencias que corresponden a un patrón de estratificación continua. Los jóvenes usan esta forma en el $23 \%$ de los casos mientras que los hablantes adultos lo usan el $14 \%$ y únicamente el $9 \%$ en los hablantes mayores.

\begin{tabular}{|l|c|c|c|}
\hline & Joven & Adulto & Mayor \\
\hline Así & $23.7 \%(41)$ & $13.75 \%(22)$ & $8.75 \%(7)$ \\
\hline SER de que & $18.5 \%(32)$ & $2.5 \%(4)$ & $2.5 \%(2)$ \\
\hline (Y)FN & $24.86 \%(43)$ & $22.50 \%(36)$ & $22.50 \%(18)$ \\
\hline O sea & $6.94 \%(12)$ & $.63 \%(1)$ & $0 \%(0)$ \\
\hline Otros & $26 \%(45)$ & $60.63 \%(97)$ & $66.25 \%(53)$ \\
\hline \multicolumn{1}{|c|}{ Total } & $100 \%(173)$ & $100 \%(160)$ & $100 \%(80)$ \\
\hline
\end{tabular}

Cuadro 8. Distribución de marcadores no canónicos por grupo de edad 
La distribución de los usos del marcador 'SER de que' revela que es una forma altamente preferida por los hablantes jóvenes $(18.5 \%)$ y prácticamente inexistente en los hablantes mayores y adultos (2.5\% en ambos grupos). Un patrón similar se observa en el caso del marcador 'o sea', cuyo uso predominante se encuentra en los hablantes jóvenes (6.94\%), y es prácticamente inexistente en los adultos (.63\%) y no aparece en los mayores (0\%). En el caso de este introductor las frecuencias y los patrones de distribución por grupo de edad sugieren que también está socialmente estratificada.

Los resultados para el marcador 'así', muestran que son los hablantes jóvenes quienes más lo usan (23.7\%) seguidos por los adultos (13.75\%) y, finalmente, el grupo de hablantes mayores (8.75\%). En el caso de la forma Y(FN) las distribuciones de porcentajes son similares entre los tres grupos: $24.86 \%$ para los hablantes jóvenes, $22.50 \%$ para los hablantes adultos y $22.50 \%$ para los mayores. Como se mencionó anteriormente, esta es la única forma no canónica en la que jóvenes, adultos y mayores comparten patrones de uso y frecuencias. Este resultado contrasta con los datos obtenidos por Cameron (1998) en los que muestra que la forma $\mathrm{Y}(\mathrm{NP})$ en el español de Puerto Rico es desfavorecida por los hablantes mayores, pero en el caso del español de México no fue este el caso.

El grupo de marcadores de la categoría 'otros' muestra una distribución etaria interesante ya que es la forma no canónica que predomina en los hablantes adultos y mayores. En el caso de los adultos, los marcadores 'otros' corresponden al 61\% del total de todos los marcadores no canónicos, y en el caso de los mayores, el uso asciende a $66 \%$. En el caso de los hablantes jóvenes, la frecuencia de estos marcadores corresponde únicamente al $26 \%$, lo cual no es muy diferente a la frecuencia de 'así' y ' $\mathrm{Y}(\mathrm{FN})$ '. Estos resultados sugieren que las formas no canónicas son muy variadas y frecuentes en el habla joven, y en el caso del habla adulta, las formas no canónicas son predominantemente aquellas del grupo 'otros.4

\footnotetext{
${ }^{4}$ El grupo de 'otros' está compuesto en gran parte por verbos de movimiento como
} 


\subsubsection{Marcadores no canónicos por nivel educativo}

El cuadro 9 ilustra la distribución y porcentajes de uso de los introductores de citas no canónicos cruzados con el nivel educativo. Los datos muestran diferencias importantes entre los usos preferidos por los hablantes de cada uno de estos niveles. En primer lugar, se observa que en el caso de los hablantes de nivel alto hay un uso considerable de las cinco formas innovadoras, siendo la forma 'o sea' la de menor frecuencia.

\begin{tabular}{|l|c|c|c|}
\hline & Alto & Medio & Bajo \\
\hline Así & $25 \%(37)$ & $16 \%(23)$ & $8 \%(10)$ \\
\hline SER de que & $15 \%(23)$ & $7 \%(10)$ & $4 \%(5)$ \\
\hline Y)FN & $35 \%(52)$ & $9 \%(13)$ & $27 \%(32)$ \\
\hline O sea & $7 \%(10)$ & $1 \%(2)$ & $1 \%(1)$ \\
\hline Otros & $18 \%(27)$ & $67 \%(96)$ & $60 \%(72)$ \\
\hline & $100 \%(149)$ & $100 \%(144)$ & $100 \%(120)$ \\
\hline
\end{tabular}

Cuadro 9. Distribución de marcadores no canónicos por nivel educativo

Ninguna de las formas no canónicas representa más del $50 \%$ en el nivel alto a diferencia del nivel medio y bajo en los que hay una marcada preferencia por otros marcadores correspondiente a la categoría "otros". Los resultados muestran que su uso predomina en los hablantes de nivel medio, con $67 \%$ de los ejemplos y $60 \%$ para el nivel bajo. Estos porcentajes contrastan con los hablantes de nivel alto que utilizan los otros marcadores en el $18 \%$ de los ejemplos.

El marcador 'así' se presenta con mayor frecuencia en los hablantes de nivel alto (25\%) seguido por los hablantes de nivel medio (16\%) y, finalmente, los hablantes de nivel bajo (8\%). El marcador 'SER de que' muestra un patrón similar, aunque frecuencias diferentes; predomina su uso en los hablantes de nivel alto (15\%) seguido por el nivel medio (7\%) y, finalmente, el nivel

'llegar', 'empezar' y por marcadores discursivos como 'este', entre otras formas. Sin embargo, será interesante ver en futuros trabajos si al interior del grupo 'otros' la distribución de cada una de las formas que componen esta categoría varía en función de las variables sociales. 
bajo (4\%). Tanto en el caso del marcador 'así' como en el caso de 'SER de que' se observa un patrón de estratificación continua.

La forma (Y)FN muestra una distribución diferente; predomina en el nivel alto (35\%) seguido por el nivel bajo (27\%) y, finalmente, el nivel medio (9\%).

La distribución del marcador 'o sea' muestra un patrón muy parecido cuando se compara el nivel educativo con la edad. En el caso del nivel educativo, esta forma predomina en el nivel alto (7\%), seguida por el nivel medio y bajo, en donde es prácticamente inexistente, $1 \%$ en cada grupo.

Los patrones de distribución de la mayor parte de las formas no canónicas sugieren que estas son preferidas por hablantes con un nivel educativo alto, ya que es este grupo el que tiene las frecuencias de uso mayores. Asimismo, si se considera el número total de marcadores que aparece por nivel educativo, es el nivel alto el que tiene proporcionalmente un porcentaje mayor de marcadores no canónicos: 12\% (149/1233) para el nivel alto, 9\% (144/1538) para el nivel medio, y 6\% (120/1861) para el nivel bajo.

\subsubsection{Marcadores no canónicos por sexo}

El análisis de la distribución de los marcadores no canónicos por sexo muestra diferencias importantes entre las frecuencias de uso de hombres y mujeres. En el caso de marcador 'así, la frecuencia de uso en las mujeres $(22 \%)$ es tres veces mayor que en los hombres (7\%), es decir, que las formas 'así que de', 'así que' son preferidas por las mujeres. En el caso del marcador 'SER de que’ el patrón es inverso; este marcador es preferido por los hombres (15\%) y utilizado con poca frecuencia por las mujeres (6\%). La diferencia de uso entre mujeres y hombres es más del doble.

\begin{tabular}{|l|c|c|}
\hline & Mujeres & Hombres \\
\hline Así & $22 \%(61)$ & $7 \%(9)$ \\
\hline SER de que & $6 \%(17)$ & $15 \%(21)$ \\
\hline (Y)FN & $28 \%(76)$ & $15 \%(21)$ \\
\hline O sea & $1 \%(4)$ & $7 \%(9)$ \\
\hline Otros & $43 \%(118)$ & $56 \%(77)$ \\
\hline & $100 \%(276)$ & $100 \%(137)$ \\
\hline
\end{tabular}

Cuadro 10. Distribución de los marcadores no canónicos por sexo 
El uso del marcador $\mathrm{Y}(\mathrm{FN})$ en las mujeres (28\%) supera a su uso en los hombres (15\%), la diferencia de casi el doble entre ambos sexos sugiere que esta forma es un marcador asociado al habla femenina. Por el contrario, la distribución del marcador 'o sea' muestra una preferencia de uso por los hombres (7\%) y muy poco uso en el caso de las mujeres (1\%).

Finalmente, la distribución de los marcadores 'otros' muestra una distribución más balanceada que el resto de los marcadores, ya que en el caso de las mujeres su uso corresponde al $43 \%$ de los datos y en el de los hombres al 56\%.

En resumen, esta sección muestra que los marcadores 'así' y '(Y)FN' son las formas no canónicas preferidas por las mujeres, mientras que los marcadores 'SER de que' y 'o sea' parecen estar más asociados al habla masculina. En el caso de los otros marcadores no parece haber ningún patrón de preferencia por género.

\section{Análisis de resultados}

A pesar de que el análisis presentado en este trabajo está basado únicamente en frecuencias y porcentajes, y que las generalizaciones deben considerarse por el momento como patrones de distribución únicamente en función de las frecuencias de uso, hay resultados importantes que pudieran ser indicadores de cambio lingüístico en unos casos y de variación estable en otros. Asimismo, los resultados encontrados muestran usos de formas que no habían sido documentadas anteriormente en el español de México y también muestran resultados muy similares particularmente a los del español de Puerto Rico (Cameron, 1998).

En términos generales, se observa que al igual que en el resto de los dialectos del español, el marcador dicendi sigue siendo la forma que más se usa para introducir citas directas en el español de México, seguido por el 'marcador nulo'. Los datos previos del español de México y de otras variedades dialectales, muestran porcentajes muy similares en sincronía y diacronía (cuadro 11). Esto sugiere que, a pesar de la incursión de nuevos marcadores, las formas dicendi se han mantenido bastante estables en el español a lo largo del tiempo y el espacio. 


\begin{tabular}{|l|c|c|c|c|c|}
\hline & $\begin{array}{c}\text { Puerto Rico } \\
\text { (Cameron, } \\
1998)\end{array}$ & $\begin{array}{c}\text { Venezuela } \\
\text { (Gallucci } \\
\text { y Vargas, } \\
2015)\end{array}$ & $\begin{array}{c}\text { España } \\
\text { (Estellés- } \\
\text { Arguedas, } \\
2015)\end{array}$ & $\begin{array}{c}\text { México } \\
1967 \text { a 1975 } \\
\text { (van der } \\
\text { Houwen, 1998) }\end{array}$ & $\begin{array}{c}\text { México } \\
(1995 \mathrm{a} \\
2005)\end{array}$ \\
\hline Dicendi & $62 \%$ & $53 \%$ & $71 \%$ & $75 \%$ & $63 \%$ \\
\hline Nulo & $25 \%$ & $40 \%$ & $16 \%$ & -- & $28 \%$ \\
\hline (Y)FN & $13 \%$ & $4 \%$ & -- & -- & $2 \%$ \\
\hline Así & -- & -- & -- & -- & $2 \%$ \\
\hline Otro & -- & $3 \%$ & $13 \%$ & $25 \%$ & $5 \%$ \\
\hline Total & $100 \%$ & $100 \%$ & $100 \%$ & $100 \%$ & $100 \%$ \\
\hline
\end{tabular}

Cuadro 11. Comparación dialectal de los usos de marcadores de citas directas

La alta y constante frecuencia de uso de las formas dicendi en el español contrasta con los datos del inglés. En esta lengua, los jóvenes utilizan en su mayoría las formas innovadoras go, be like, be all, para introducir citas directas, y en mucho menor medida los verbos dicendi (Ferrara y Bell, 1995; Tagliamonte y Hudson, 1999; Tagliamonte y D’Arcy, 2004; Buchstaller y D’Arcy, 2009) y en el caso del español de México, las formas no canónicas, que muy probablemente sean formas innovadoras, corresponden únicamente al $9 \%$ de los datos. Es importante mencionar que la distribución tan similar de los marcadores dicendi y nulo en los dialectos del español previamente estudiados (cuadro 11) sugiere que, de haber un cambio lingüístico en proceso, este está ocurriendo en el resto de los marcadores, y las formas canónicas parecen ser formas sumamente estables en el sistema de citas directas del español. Es necesario investigar en futuros trabajos si en las formas dicendi del español contemporáneo predomina el uso del presente sobre el pretérito, como está documentado en el español de los sesenta (van der Houwen, 1998).

En el caso particular de los marcadores dicendi se observa con mayor frecuencia en hablantes de nivel bajo y en mujeres. Los marcadores nulos como estrategia para introducir citas directas en el español de México son formas más comunes en los hombres y en nivel alto. Este mismo patrón en el que las mujeres prefieren la forma dicendi y los hombres el marcador nulo ha sido documentado también en el español de Venezuela (Galluc- 
ci y Vargas, 2015). Con respecto al nivel educativo y los usos de marcadores canónicos, el patrón del español de México aquí reportado es exactamente igual al que encontró Cameron (1998); los verbos dicendi son más frecuentes en hablantes de nivel bajo y los 'marcadores nulos' son más frecuentes en los hablantes de nivel alto. Lo mismo ocurrió en el caso del género; tanto en el español de Puerto Rico como en el español de la ciudad de México, el marcador nulo fue preferido por hombres y poco utilizado por las mujeres.

Finalmente, cabe mencionar que en el caso de ambos marcadores canónicos su función se restringe al reporte directo de un acto de habla, lo que sugiere que los marcadores innovadores tienen funciones alternativas como las reportadas por la forma be like, que inicialmente fue identificada como una manera de decir pensamientos, puntos de vista o monólogos internos, pero que con el tiempo se extendió al reporte de actos de habla:

Hence, the available evidence suggests that be like, when it first emerged, predominantly framed thoughts, stances and inner monologues within the English quotative system, but it quickly broadened its remit to introduce quotations of both speech and thought reports. In present-day usage, be like is ambiguous as regards the outward occurrence of the quote (Buchstaller, 2013: 8).

Queda por investigar si en el caso del español de México, y de otros dialectos, las formas innovadoras tienen funciones discursivas particulares y si han seguido el patrón de incursión en el sistema que se ha observado en otras lenguas, en el que inicialmente tienen una función especializada y después se observa extensión a otros dominios.

Los resultados sobre la frecuencia y la distribución de las formas no canónicas sugieren que están socialmente estratificadas (mayor frecuencia jóvenes, nivel alto y mujeres). Los patrones de distribución de las formas no canónicas por edad, particularmente el caso de 'SER de que' y 'o sea', además de mostrar la estratificación etaria de las formas de citas directas no canónicas, sugieren que podríamos estar ante un cambio en progreso como 
se ha mostrado en el inglés. El marcador 'asî' es más frecuente en mujeres, jóvenes, con nivel de instrucción alto, y el marcador 'SER de que' predomina en hombres, jóvenes, con nivel de instrucción alto. En el caso del marcador (Y) FN no se observa estratificación por edad, sin embargo, es preferido por mujeres hablantes de nivel alto. Este resultado nuevamente coincide con lo reportado por Cameron (1998) para el español puertorriqueño, en el que la forma (Y)FN es favorecida por las mujeres y desfavorecida por los hombres. El marcador 'o sea' muestra un claro patrón de estratificación por nivel de edad, nivel de instrucción y género, dicha forma es preferida por hombres jóvenes, de nivel alto. Finalmente, los marcadores clasificados como 'otros' son preferidos por hablantes adultos y mayores, de nivel medio y bajo, sin distinción por género.

\section{Conclusiones}

El análisis descriptivo de las estrategias introductoras de citas directas en el español de México muestra que este sistema está conformado por marcadores canónicos (dicendi y nulo), así como por marcadores no canónicos (innovadores). Estos datos son compatibles con los estudios previos que han mostrado que el sistema de citas directas es un sistema dinámico y en el que se observa constante innovación. Si bien en este trabajo únicamente se presenta un análisis descriptivo, estos resultados sugieren también que el sistema de citas directas en el español de México, al igual que el de otras variedades dialectales y al igual que otras lenguas, está socialmente estratificado. De la misma manera, los datos sugieren una división del sistema; mientras las formas canónicas (dicendi y marcador nulo) parecen estar en variación estable, las formas no canónicas o innovadoras muestran mayor evidencia de cambio en progreso y patrones de estratificación más claros.

Resulta necesario indagar en el desarrollo diacrónico de los marcadores de citas directas en el español de México, especialmente en las formas no canónicas, de las que aparentemente no hay registro en los estudios previos. 
Si bien es necesario dar el siguiente paso en el análisis para determinar la interacción de los factores internos y externos en la variación y el cambio en el sistema de citas directas del español, podemos afirmar, por ahora, que los patrones de uso que muestran los datos sugieren que el sistema de citas del español de México es un sistema socialmente estratificado que está en proceso de cambio lingüístico y que nuevas formas se están incorporando.

\section{Bibliografía}

Briz, A. (2006), "La segmentación de una conversación en diálogos”, Oralia: Análisis del Discurso Oral, 9, pp. 45-72.

Buchstaller, I. (2013), Quotatives: New trends and sociolinguistic implications, Oxford, John Wiley y Sons.

Buchstaller, I. y A. D’Arcy (2009), "Localized globalization: A multi-local, multivariate investigation of quotative be like", Journal of Sociolinguistics, 13:3, pp. 291-331.

Buchstaller, I., J. R. Rickford, E. C. Traugott, T. Wasow y A. ZwiCKy (2010), “The sociolinguistics of a short-lived innovation: Tracing the development of quotative all across spoken and internet newsgroup data", Language Variation and Change, 22:2, pp. 191-219.

Cabedo Nebot, A. (2007), "Caracterización prosódica del estilo directo de habla en la conversación coloquial", ELUA, 21, pp. 53-64.

Camargo Fernández, L. (2007-2008), “La cita como turno: el diálogo reconstruido en español oral desde la pragmática de corpus", Pragmalingüística, 15-16, pp. 49-70.

Camargo Fernández, L. (2012), "La representación del discurso en los corpus PRESEEA. Metodología y propuesta de análisis”, < <ttp://preseea.linguas.net/Metodolog\%C3\%ADa. aspx $>$ [consultado en diferentes momentos].

Cameron, R. (1998), "A variable syntax of speech, gesture, and sound effect: Direct quotations in Spanish", Language Variation and Change, 10:1, pp. 43-83. 
Clark, H. H., y R. J. Gerrig (1990), “Quotations as demonstrations", Language, pp. 764-805.

Estellés-Arguedas, M. (2015), “Expressing evidentiality through prosody? Prosodic voicing in reported speech in Spanish colloquial conversations", Journal of Pragmatics, 85, pp. 138-154.

Fernández Moreno, F. (2012), "Discurso directo e indirecto en el español de Mérida”, Lengua y Habla, 16:1, pp. 71-85.

Ferrara, K. y B. Bell (1995), "Sociolinguistic variation and discourse function of constructed dialogue introducers: The case of be+ like", American Speech, 70:3, pp. 265-290.

Gallucci, M. J. (2009), “Nos fuimos a la casa, y mi mamá: ¿estaban lanzándose por la quebrada?, ¡no, mamá!, ¡claro que sí!: estudio piloto de las citas conversacionales en el habla de Caracas", Núcleo, 26, pp. 75-98.

GALlucci, M. J. (2012a), "Estilo directo e indirecto en interacciones orales. Estado de la cuestión en el ámbito hispánico", Boletín de Filología, XLVII:2, pp. 205-233.

Gallucci, M. J. (2012b), "Sintaxis de las citas en estilo directo e indirecto con verbo en el habla caraqueña”, Lingüística, 28, pp. 223-246.

GALluCCI, M. J. (2013), “Más sobre el estilo directo e indirecto en el español de Caracas”, Lengua y Habla, 17:1, pp. 89-117.

Gallucci, M. J. (2014), "Entonces un muchacho llega y me dice: 'Mira, regáleme un bolívar'. Las citas en estilo directo e indirecto en el Corpus diacrónico del habla de Caracas 1987/2013”, Boletín de Lingüística, XXVI:41-42, pp. 45-74.

Gallucci, M. J. y K. Vargas (2015), "Estilo directo e indirecto en el Corpus sociolingüístico de Caracas 1987”, Anuario de Letras. Lingüística y Filología, III:2, pp. 65-104.

GARcíA, R. (2009), "Aspectos pragmáticos y funcionales del discurso referido en la interacción cara a cara", tesis de licenciatura, Puebla, Benemérita Universidad Autónoma de Puebla.

Holguín Mendoza, C. (2015), "Pragmatic functions and cultural communicative needs in the use of 'y yo' and 'así' ('be+ like') among Mexican bilingual youth", en K. Potowski y T. 
Bugel (eds.), Papers in Honor of Anna María Escobar's anniversary at the University of Illinois at Urbana Champaign, Nueva York, Peter Lang., pp. 57-92.

Houwen, F. VAN Der (1998), "Organizing discourse. Direct and indirect speech in Mexican Spanish", Linguistics in the Netherlands, 15, pp. 123-134.

KvaviK, K. H. (1986), "Characteristics of direct and reported speech prosody: Evidence from Spanish", en F. Coulmas (ed.), Direct and Indirect Speech, La Haya, Mouton de Gruyter, pp. 333-360.

Labov, W. (1963), "The social motivation of a sound change", Word, 19:3, pp. 273-309.

Laвоv, W. (1966), The social stratification of English in New York City, Washington, Center for Applied Linguistics.

Maldonado, C. (1999), "Discurso directo y discurso indirecto", en I. Bosque y V. Demonte (dirs.), Gramática descriptiva de la lengua española, Madrid, Espasa Calpe, vol. 3, pp. 35493596.

Martín Butragueño, P. y Y. Lastra (coords.) (2011), Corpus sociolingüístico de la ciudad de México (3 vols.), México, El Colegio de México.

Maschler, Y. (2002), “On the grammaticization of ke'ilu 'like, lit. 'as if', in Hebrew talk-in-interaction", Language in Society, 31:2, pp. 243-276.

Mateus, L. (2005), "El estilo directo e indirecto como estrategias narrativas en el habla de Caracas", tesis de licenciatura, Caracas, Universidad Central de Venezuela.

Moreno Fernández, F. (1997), "Metodología del proyecto sociolingüístico para el estudio del español de España y de América (PRESEEA)", en F. Moreno Fernández (ed.), Trabajos de sociolingüística hispánica, Alcalá de Henares, Universidad de Alcalá, pp. 137-166.

Moreno Fernández, F. (2003), "Metodología del proyecto sociolingüístico para el estudio del español de España y de América (PRESEEA)" (versión revisada, octubre 2003). <http://preseea.linguas.net/Metodolog\%C3\%ADa.aspx> [consultado en diferentes momentos]. 
Palacios Martínez, I. M. (2013), "Zero quoting in the speech of British and Spanish teenagers: A contrastive corpus-based study", Discourse Studies, 15:4, pp. 439-462.

Romaine, S. y D. LANGe (1991), "The use of like as a marker of reported speech and thought: A case of grammaticalization in progress", American Speech, 66:3, pp. 227-279.

Tagliamonte, S. y A. D’Arcy (2004), "He’s like, she's like: The quotative system in Canadian youth", Journal of Sociolinguistics, 8:4, pp. 493-514.

Tagliamonte, S. y R. Hudson (1999), "Be like et al. beyond America: The quotative system in British and Canadian youth", Journal of Sociolinguistics, 3:2, pp. 147-172.

TAnnen, D. (1986), "Introducing constructed dialogue in Greek and American conversational and literary narrative", Direct and Indirect Speech, 31, pp. 311-332.

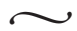

Juliana De la Mora Gutiérrez ( $\mathrm{PhD}$ The Ohio State University) es Profesora de Tiempo Completo en la Universidad Autónoma de Querétaro y miembro del Sistema Nacional de Investigadores. Sus principales líneas de investigación son la variación pragmático-discursiva y la sociolingüística. Actualmente dirige el proyecto "Sincronía y diacronía de las marcas de citas en el español de México". 